\title{
Recursos Educacionais Abertos para o Ensino e Aprendizado de Programação no Brasil: Primeiros Referenciais
}

\author{
William Simão de Deus ${ }^{1}$, Ellen Francine Barbosa ${ }^{1}$ \\ ${ }^{1}$ Instituto de Ciências Matemáticas e de Computação (ICMC) \\ Universidade de São Paulo (USP) \\ Av. Trab. São Carlense, 400 - Centro, São Carlos - SP, 13566-590 \\ williamsimao@usp.br, francinedicmc.usp.br
}

\begin{abstract}
This study presents an overview of the Open Educational Resources (OERs) for teaching and learning programming in Brazil. Using an exploratory case study, 37 OERs were collected and analyzed in order to provide the first references on the subject. The study presents a summary of resources, in addition to debating the level of openness and identifying the main producers. Despite the promising data, the results point to problems of these resources, such as proprietary formats and lack of search mechanisms.
\end{abstract}

Resumo. Este estudo apresenta um panorama geral sobre os Recursos Educacionais Abertos (REAs) para o ensino e aprendizado de programação no Brasil. Por meio de um estudo de caso exploratório, foram coletados e analisados 37 REAs a fim de fornecer os primeiros referenciais sobre o assunto. O estudo apresenta um resumo dos recursos, além de debater o nível de abertura e identificar os principais produtores. Apesar dos dados promissores, os resultados apontam os problemas dos recursos, como formatos proprietários e falta de mecanismos de buscas.

\section{Introdução}

Recursos Educacionais Abertos (REAs) são materiais voltados ao ensino, aprendizagem ou pesquisa que podem ser acessados, utilizados ou até mesmo adaptados por outros usuários [Amiel et al. 2019]. No contexto educacional, REAs permitem que alunos ou professores utilizem diversos tipos de materiais, como slides, vídeos, exercícios ou outros recursos, ao mesmo tempo em que reduzem problemas de direitos autorais.

No ensino e aprendizado de programação a adoção de REAs vem ganhando cada vez mais atenção. [Chan et al. 2020], por exemplo, analisaram uma das principais comunidades abertas de Ciência da Computação e perceberam que a maioria dos materiais são referentes à programação. De modo geral, alunos e professores estão cientes dos benefícios de utilizarem tais recursos. Como [Baldwin 2015] relatou, ao projetar um curso introdutório de programação usando apenas REAs disponíveis na Internet, o autor percebeu uma série de benefícios, como a economia do tempo (já que os materiais estavam disponíveis) e a redução de custos (pois os recursos reusados eram gratuitos).

No Brasil é possível identificar uma série de iniciativas públicas que buscam promover o uso de REAs, como repositórios abertos lançados por universidades públicas, criadores independentes, projetos de lei e ações da sociedade civil [Amiel et al. 2018]. Mesmo assim, apesar do potencial de mudança e da experiência brasileira sobre REA, 
ainda não existem dados que forneçam uma visão geral sobre os REAs de programação no território nacional.

A maioria dos estudos atuais que analisam os REAs para ensino e aprendizado de programação são concentrados em iniciativas internacionais, focando materiais disponíveis em inglês, como [Chan et al. 2020] e [Dichev and Dicheva 2012]. Por isso, não é possível generalizar os resultados obtidos. Ao mesmo tempo, o ensino e aprendizado de programação é um instrumento relevante para a formação do pensamento computacional [Araujo et al. 2016], sendo essencial entender o papel destes materiais e o potencial que eles possuem para auxiliar a rotina dos alunos e professores em todo o Brasil.

Considerando este cenário, o objetivo deste estudo é apresentar os primeiros referenciais sobre os REAs voltados ao ensino e aprendizado de programação em território nacional. Para isso, foi conduzido um estudo de caso exploratório em um dos principais portais de ensino no Brasil. Foram analisados conteúdos dos materiais, além de detalhar a abertura dos recursos e quem vem produzindo e disponibilizando REAs. Com base nos resultados percebe-se que apesar do grande potencial de apoio na rotina de alunos e professores, os REAs de programação ainda possuem desafios a serem superados, como falta de dados de identificação e mecanismos de recuperação dos recursos.

O restante deste estudo encontra-se organizado da seguinte forma: A fundamentação teórica sobre REAs e ensino de programação é apresentada na Seção 2. O método de investigação é sumarizado na Seção 3. Os resultados obtidos são apresentados na Seção 4 e as discussões na Seção 5 . Por fim, as limitações, trabalhos futuros e considerações finais estão apresentadas na Seção 6 .

\section{Fundamentação Teórica}

Um REA pode assumir qualquer formato e por isso mesmo é muito comum ser encontrado em diferentes sites e portais de ensino. No entanto, para um material ser considerado um REA é essencial que ele possua um propósito educacional e que ele seja aberto.

O propósito educacional refere-se à capacidade do recurso apoiar o ensino, o aprendizado ou a pesquisa de um tema. Já a a abertura de um REA é o principal elemento para que outros alunos e professores reusem ou adaptem o material. De acordo com a [OECD 2007] existem três domínios de abertura nos REAs: O primeiro é a abertura técnica, que se refere ao uso de padrões e softwares abertos para que o material possa ser usado e editado facilmente sem custos ou barreiras. Um exemplo desse domínio de abertura é a adoção do formato .odt ou .html ao invés do .pdf ou .docx para livros digitais. O segundo domínio refere-se à abertura social. Neste caso, o REA deve ser projetado considerando que o maior número de pessoas na sociedade possa usá-lo, como por exemplo alunos com restrições de acessibilidade. Por fim, existe a abertura como característica, consistindo na necessidade de projetar o REA para que outras pessoas criem novos REAs. Por exemplo, projetar um livro digital com capítulos que podem ser transformados em novos materiais, como videoaulas, podcasts ou listas de exercícios.

Quando se fala em REAs para o ensino de programação é comum lembrar dos projetos de software livre. Tais projetos possuem muitas das premissas sobre abertura destacadas anteriormente. Mas, conforme comentado, para ser um REA é necessário que o recurso possua um propósito educacional. Ou seja, não basta ser aberto, é essencial 
que seu conteúdo possa auxiliar o processo educacional. Por isso mesmo, muitos autores classificam projetos de software livre como um domínio diferente dos REAs. De fato, projetos de software livre são excelentes ferramentas para a criação e edição de REAs [OECD 2007], já que na maioria das vezes não oferecem barreiras de acesso. Por conta disso, os REAs voltados ao ensino e aprendizado de programação explorados neste estudo são materiais educacionais, como livros digitais, cursos e ferramentas, projetados para apoiar a rotina de alunos e professores de programação. Além disso, são materiais abertos, que fomentam e auxiliam a cultura aberta, como a possibilidade de revisar, adaptar ou criar novos materiais derivados de recursos existentes, diferentemente dos objetos fixos de aprendizagem. Até o melhor do nosso conhecimento, não existe uma pesquisa analisando tal perspectiva no cenário brasileiro, como detalhado a seguir.

\subsection{Trabalhos Relacionados}

Atualmente, a literatura concentra estudos com propósitos similares ao apresentado nesta investigação. Um exemplo é o trabalho desenvolvido por [Chan et al. 2020] que analisou 100 REAs de uma comunidade aberta de Ciência da Computação. As principais diferenças entre este estudo e o estudo de [Chan et al. 2020] é que os autores limitaram a investigação nos recursos mais usados e realizaram uma busca automática. No estudo aqui apresentado, todos os REAs retornados foram analisados. Além do mais, os recursos foram analisados manualmente, reduzindo o viés de buscas não supervisionadas.

Outro estudo que fez uma investigação similar à apresentada neste trabalho foi conduzida por [Dichev and Dicheva 2012]. Nele, os autores coletaram respostas de usuários e buscaram por REAs voltados ao ensino de Ciência da Computação, identificando 8 recursos relacionados a programação. O trabalho aqui exposto e o de [Dichev and Dicheva 2012] diferem-se porque os autores analisaram um conjunto menor de REAs de programação. Além disso, os conceitos de programação explorados pelos estudos são distintos.

Por fim, [Chan et al. 2020] e [Dichev and Dicheva 2012] não analisaram recursos disponíveis no idioma português, sendo esta a principal diferença entre os trabalhos relacionados e o estudo aqui relatado.

\section{Design do Estudo de Caso}

Esta investigação foi baseada nas premissas de [Filho et al. 2021] sobre a execução de estudos de caso em Informática na Educação. A seguir, as principais etapas do método de pesquisa são sumarizadas.

\subsection{Formulação do Problema}

O problema investigado neste estudo refere-se à falta de dados sobre os REAs para ao ensino e aprendizado de programação no Brasil. A fim de fornecer os primeiros referenciais sobre o tema, três Questões de Pesquisa (QP) foram formuladas:

- $\mathrm{QP}_{1}$ ) Qual é o conteúdo dos REAs de programação no Brasil?

- $\mathrm{QP}_{2}$ ) Qual é a abertura dos REAs de programação no Brasil?

- $\mathrm{QP}_{3}$ ) Quem produz e disponibiliza REAs de programação no Brasil? 


\subsection{Seleção do Caso}

Para solucionar a QPs previamente estabelecidas, foi iniciado o procedimento de busca e identificação de um caso relevante. Em primeiro lugar, considerando o escopo de investigação, foram considerados apenas casos brasileiros. Por isso iniciativas estrangeiras de REAs não foram consultadas. Em segundo lugar, o caso deveria possuir REAs voltados ao ensino e aprendizado de programação. Para isso, potenciais casos relevantes foram acessados pelos autores a fim de examinar o acervo. Por fim, o caso deveria possuir uma estrutura capaz de solucionar as QPs estabelecidas.

Após analisar potenciais casos de análise, o portal EduCapes ${ }^{1}$ foi selecionado. O portal atendeu a todos os critérios estabelecidos e foi considerado o caso a ser analisado tendo em vista que fornecia uma estrutura padrão para recuperar informações dos REAs, além de fornecer insumos para a solução das QPs estabelecidas. De modo resumido, o portal EduCapes é voltado aos alunos e professores de diferentes níveis educacionais no Brasil, possui um acervo que inclui textos, livros, artigos, videoaulas e outros tipos de recursos educacionais. Além disso, o EduCapes lista recursos de outros repositórios nacionais. Por fim, o portal utiliza um esquema de metadados que permite recuperar informações estruturadas para responder as QPs realizadas neste estudo.

\subsection{Coleta de Dados}

O portal EduCapes possui um acervo dinâmico. Isto é, sua coleção é organizada por meio de palavras-chaves usadas nos recursos. Por isso não existe uma forma padronizada de extrair apenas os REAs voltados ao ensino e aprendizado de programação. Pode ser que um recurso seja identificado pelo termo "Programação " enquanto outro esteja sob o termo "Programação de Computadores ". Considerando este cenário, foi elaborado um conjunto de quatro etapas ${ }^{2}$, para realizar tal procedimento a fim de se extrair o maior número de REAs relevantes para análise.

Etapa 1: Foi feita uma busca por meio da string "programação" e um algoritmo programado pelos autores extraiu os resultados retornados em uma planilha eletrônica. Neste estágio, 3.715 resultados foram recuperados.

Etapa 2: Os resultados foram filtrados automaticamente a fim de selecionar apenas materiais com licença aberta e no idioma português, que são as premissas deste estudo. Ao aplicar este filtro, 1.669 resultados foram selecionados.

Etapa 3: Foi criado um novo filtro automático para remover resultados não relevantes. Foi gerada uma lista de práticas de programação de computadores adotadas por alunos e professores no Brasil, conforme o mapeamento de [Araujo et al. 2016]. Ao final desta etapa, 174 REAs foram selecionados.

Etapa 4: Após análise dos autores, percebeu-se a presença de recursos não relevantes para investigação, como recursos que usavam uma linguagem de programação para construir um projeto. No entanto, não havia foco no ensino ou no aprendizado sobre os conceitos de programação de computadores. Por isso, os autores revisaram manualmente os recursos e selecionaram 37 REAs para análise.

\footnotetext{
1'https://educapes.capes.gov.br/

${ }^{2}$ Os códigos e dados estão disponíveis no seguinte link: https://doi.org/10.5281/zenodo.5510506
} 


\section{Primeiros Referenciais sobre os REAs para Programação no Brasil}

Com base no conjunto de dados formado pelos recursos coletados foi iniciada a análise das QPs deste estudo. A Tabela 1 apresenta uma lista dos recursos selecionados.

Tabela 1: Recursos selecionados para análise.

\begin{tabular}{|c|c|c|}
\hline Número & Recurso Educacional Aberto e Link de Acesso & Área de Conhecimento \\
\hline 01 & Aprenda Programação Gratuitamente & Tecnologia \\
\hline 02 & Projeto De Scratch - Aprendendo A Reciclar & Programação/Informática \\
\hline 03 & Introdução A Programação De Computadores & \\
\hline 04 & Curso Básico De Programação De Computadores Para Adolescentes & Prática de Pesquisa Orientada \\
\hline 05 & Introdução À Programação C++ & \\
\hline 06 & Introdução A Linguagem De Programação Delphi & \\
\hline 07 & Oficina De Programação De Computadores Para Crianças - 2A. Edição & \\
\hline 08 & Estrutura Condicional If & Introdução à Programação \\
\hline 09 & Introdução A Linguagem C & Introdução à Computação \\
\hline 10 & Desenvolvimento De Aplicativos Móveis Com Xamarin Forms & Educação Aberta \\
\hline 11 & $\begin{array}{l}\text { Guia Para A Criação De Objetos De Aprendizagem Personalizados No Ambiente } \\
\text { Adaptweb }\end{array}$ & Informática - Ensino Superior \\
\hline 12 & Framework Gamificado Para Disciplina Algoritmos Ou Equivalentes & Ensino \\
\hline 13 & Metodologia 7Cs: Material De Orientações & Ensino \\
\hline 14 & Infográfico - O Que E Pensamento Computacional? & REAs* \\
\hline 15 & Expressões Lógicas - Operadores Lógicos E Tabela-Verdade & Lógica \\
\hline 16 & Algoritmo E Estrutura De Dados 1 & Alg. e Estrutura de Dados I* \\
\hline 17 & Estrutura De Dados & Estrutura de Dados \\
\hline 18 & Algoritmo E Estrutura De Dados II & Alg. e Estrutura de Dados II* \\
\hline 19 & Quiz Na Aprendizagem: Jogo, Motivação E Interação & Informática na educação* \\
\hline 20 & $\begin{array}{l}\text { Oficina De Programação De Computadores Para Crianças Projeto De Extensão Cons- } \\
\text { truindo Saberes Através Do Computador E Internet - Grupo De Trabalho Racioci- } \\
\text { nando Com O Computador }\end{array}$ & \\
\hline 21 & $\begin{array}{l}\text { Ensinar Programação Em Ambientes E-Learning: Preocupações E Propostas No } \\
\text { Ámbito Do Modelo Pedagógico Virtual Da Universidade Aberta De Portugal }\end{array}$ & \\
\hline 22 & $\begin{array}{l}\text { Estratégias para a Utilização de Jogos Digitais e Tecnologias Emergentes no ensino } \\
\text { de linguagem de programação }\end{array}$ & \\
\hline 23 & Primeiro Programa Em Linguagem C & Educação Aberta* \\
\hline 24 & Pesquisa E Ordenação De Dados & Pesq. e Orde. de Dados* \\
\hline 25 & $\begin{array}{l}\text { O Ensino E Aprendizagem Da Programação De Computadores No Ensino A } \\
\text { Distância: Uma Proposta De Instanciação Do Modelo Pedagógico Virtual Da Uni- } \\
\text { versidade Aberta }\end{array}$ & \\
\hline 26 & Caderno De Atividades Para O Ensino De Programação Utilizando O Scratch & Mestrado* \\
\hline 27 & Lógica Da Argumentação - Tabela Da Verdade & Lógica da programação \\
\hline 28 & Pensamento Computacional Com Scratch: Construindo O Jogo "Gato Voador" & Informática* \\
\hline 29 & Blog Programador Do Futuro & Comp. Ensino Fundamental \\
\hline 30 & Avaliação Pelos Pares: Um Guia Didático Para O Ensino De Lógica De Programação & Mestrado* \\
\hline 31 & $\begin{array}{l}\text { Guia Didático Para O Desenvolvimento Do Pensamento Computacional Em Estu- } \\
\text { dantes Do Ensino Fundamental Por Meio Do Software Scratch }\end{array}$ & Mestrado* \\
\hline 32 & Caderno Didático Programa Escola De Hackers & Mestrado* \\
\hline 33 & Algoritmos E Programação I & Algoritmos e Programação I \\
\hline 34 & $\begin{array}{l}\text { Avaliação Da Aprendizagem: Uma Estratégia Para A Disciplina De Lógica De } \\
\text { Programação No Ensino Médio Utilizando A Taxonomia De Bloom }\end{array}$ & \\
\hline 35 & $\begin{array}{l}\text { Videoaulas Para Apoiar O Processo Ensino-Aprendizagem Sobre Funções Da Disci- } \\
\text { plina De Algoritmos E Lógica De Programação }\end{array}$ & Computação - Informática \\
\hline 36 & Resolução De Problemas Na Prática De Ensino De Algoritmos E Programação & Prática de Ensino em Comp. I \\
\hline 37 & Introdução A Algoritmos & Introdução a Algoritmos \\
\hline
\end{tabular}

\subsection{O Conteúdo dos REAs $\left(\mathrm{QP}_{1}\right)$}

Os recursos são heterogêneos em seus formatos, tipos e conteúdos, demonstrando a amplitude de assuntos e contextos que podem ser apoiados pelo uso destes materiais. Ao mesmo tempo, no entanto, ocorre a fragmentação dos termos, dos assuntos e dos problemas que dificultam a correta classificação do recurso. Pela análise inicial, percebe-se uma grande presença de materiais voltados ao ensino introdutório de programação. Neste sentido, destacam-se REAs para apoiar o ensino e aprendizado de algoritmos, linguagens de programação e a ferramenta Scratch. 
Ao analisar informações cadastradas nos REAs são identificados os primeiros desafios. A falta de padronização ou de um vocabulário comum torna a classificação um processo quase inviável. Na maioria das vezes, os recursos são disponibilizando sob a área do conhecimento por meio de termos genéricos, como Computação ou Informática. Ou ainda, termos que não representam de fato o conteúdo ou a área do conhecimento do recurso, como Mestrado. O mesmo vale para níveis educacionais. Poucos citam o público-alvo, sejam em metadados específicos ou na própria descrição do recurso.

Aprofundando este problema, as descrições foram analisadas por meio de processamento textual. Os resumos foram adicionados em uma lista e os termos comuns foram removidos. Para este procedimento foi usada uma lista de stop words em português, excluindo termos com grande repetição que não adicionam significado ao recurso e termos que apareciam apenas $1 \mathrm{vez}$. Após o processamento, percebeu-se que grande parte dos termos usados são palavras frequentes no cenário educacional de programação, mas que não introduzem um significado de fato sobre o material. Por exemplo, os termos Programação, Ensino ou Disciplina são frequentemente adotados mas não trazem consigo uma referência sobre o conteúdo do material. Termos específicos que podem denotar a natureza do recurso ou o seu conteúdo não são frequentemente adotados. Este pode ser um problema, tendo em vista que os motores de busca são limitados e tendem a retornar resultados amplos e genéricos. A Figura 1 apresenta uma nuvem das palavras mais comuns usadas nos recursos.

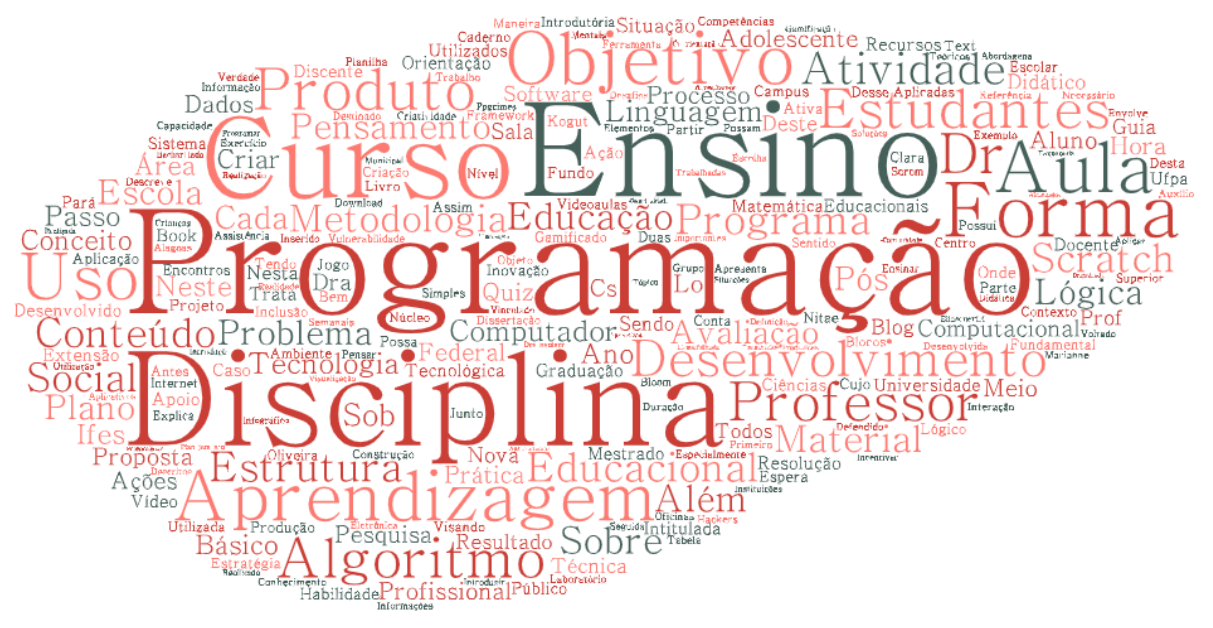

Figura 1. Termos frequentes usados na descrição dos REAs

\subsection{A Abertura dos $\operatorname{REAs}\left(\mathrm{QP}_{2}\right)$}

A abertura é um fator importante para um REA. Materiais bem projetados podem ser usados e adaptados sem oferecer limitações aos professores e alunos. Como visto na Seção 2, os REAs geralmente possuem três tipos de abertura: técnica, social e característica. Para habilitar a capacidade de criação comum entre professores e alunos é necessário que o recurso possua licenças permissivas e formatos abertos. Por isso, inicialmente, foi analisada a abertura de cada licença usando como base o framework cinco R (5'R) proposto por [Wiley 2014]. Sob a ótica deste framework, uma licença aberta pode permitir 5 níveis: Reter (o usuário pode fazer download ou cópias do material sem restrições); Reusar (o usuário pode usar o recurso para um propósito diferente do original); Revisar (o 
usuário pode revisar o material, como por exemplo, fazer uma tradução); Remixar (criar um novo recurso com base no original); Redistribuir (compartilhar o novo material para outros usuários). A Tabela 2 apresenta uma síntese dos resultados.

Tabela 2: O uso das licenças e a abertura de cada uma considerando o Framework 5'R [Wiley 2014].

\begin{tabular}{lcccccc}
\hline Licença & Reter & Reusar & Revisar & Remixar & Redistribuir & \% de REAs \\
\hline CC BY 3.0 BR & Sim & Sim & Sim & Sim & Sim & $6,67 \%$ \\
CC BY-NC 3.0 BR & Sim & Sim & Sim & Sim & Sim & $15,56 \%$ \\
CC BY-NC 4.0 & Sim & Sim & Sim & Sim & Sim & $2,22 \%$ \\
CC BY-NC-ND 3.0 BR & Sim & Sim & Sim & Não & Não & $20,00 \%$ \\
CC BY-NC-ND 4.0 & Sim & Sim & Sim & Não & Não & $2,22 \%$ \\
CC BY-NC-SA 3.0 BR & Sim & Sim & Sim & Sim & Sim & $40,00 \%$ \\
CC0 1.0 & Sim & Sim & Sim & Sim & Sim & $6,67 \%$ \\
Open Access & Sim & $?$ & $?$ & $?$ & $?$ & $6,67 \%$
\end{tabular}

Nota: O remix é aqui representado na criação de um novo recurso com base em um recuro original, por isso as licenças com clausulas ND foram classificadas como inválidas para este propósito.

A maioria dos REAs investigados estão sob a licenças Creative Commons, grande parte já adaptada para o contexto nacional (sigla BR). Isso facilita para que professores e alunos possam entender de que modo os materiais podem ser utilizados, já que os termos de uso estão em português. Ao mesmo tempo, percebe-se que a maioria das licenças permitem uma ampla abertura do recurso, enquanto 22,22\% evita práticas abertas como o remix e a redistribuição. Mesmo assim, no geral, os REAs para programação são aderentes às licenças permissivas. Encontramos alguns materiais sob a licença Open Access, no entanto, não há uma descrição sobre quais permissões tal licença oferece. Por isso, consideramos apenas a abertura mínima de uma licença, a opção de reter.

Cabe destacar que muitos materiais estão sob licenças permissivas, com cláusulas que permitem que o conteúdo seja revisado, remixado ou até redistribuído. Em muitos casos, por exemplo, o recurso está sob uma licença Creative Commons com o atributo Share a Like ou Compartilha Igual. Em suma, isso quer dizer que os usuários podem criar e distribuir materiais derivados do REA original desde que adotem uma licença com o mesmo nível de abertura. Tal prática favorece a aplicação dos REAs, a cultura do reuso e também a criação coletiva. Mas, muitos destes materiais estão em formatos proprietários, oferecendo muita resistência para criação coletiva.

Um REA pode ser composto por uma série de componentes internos. Por exemplo, um curso aberto pode ser constituído por vídeos, atividades e materiais de apoio. No entanto, é necessário que estes componentes também possuam formatos que possibilitem o reuso e a adaptação. Os 37 recursos analisados são compostos por 43 componentes internos. Desse total, apenas 30 possuem um formato especificado, sendo que: 25 REAs possuem o formato . $p d f, 2$ REAs possuem o formato . $m p 4,1$ REA possui o formato .odt, 1 recurso possui o formato .xls e 1 material possui o formato . mov.

Ou seja, observando a grande predominância de formatos fechados com licenças abertas percebe-se que muitos produtores não estão considerando a abertura técnica do material. Ao invés disso, estão usando formatos que limitam a criação comum, mesmo que forneçam licenças permissivas. Isso implica diretamente na abertura como característica do recurso, impedindo a criação de obras derivadas. Ao mesmo tempo, não identificamos nenhum elemento sobre a abertura social, como informações sobre acessibilidade. 


\subsection{Os produtores de REAs $\left(\mathrm{QP}_{3}\right)$}

Grande parte dos materiais analisados foram produzidos por instituições de ensino públicas. Programas de Pós-Graduação destacam-se como produtores de REAs voltados ao ensino e aprendizado de programação. Assim, percebe-se a disponibilização de artefatos como "produtos"ou "produtos educacionais", possibilitando a disseminação da pesquisa além de monografias e dissertações. Além disso, nota-se ainda uma grande dispersão em diferentes instituições de ensino. A Tabela 3 apresenta a listagem geral de instituições e cursos produtores de REAs.

\begin{tabular}{llc}
\multicolumn{1}{c}{ Tabela 3: Produtores de REAs de programação } & REAs \\
\hline Instituição & Curso & 3 \\
\hline UESC & Mestrado Profissional Em Ensino De Ciências, Matemática E Tecnologias & 2 \\
UECE & Computação & 2 \\
UEPG & Licenciatura Em Computação & 2 \\
UFAL & Bacharelado Em Sistemas De Informação & 2 \\
UFPA & Mestrado Profissional Em Ensino Do PPGCIMES & 2 \\
USP & Especialização Em Computação Aplicada À Educação & 1 \\
IFES & Mestrado Profissional Em Educação Profissional E Tecnológica & 1 \\
IFES & Educação Em Ciências E Matemática & 1 \\
IFSertãoPE & Programa De Pós-Graduação Em Educação Profissional E Tecnológica & 1 \\
IFSP & Técnicos, Ensino Médio, Ensino Fundamental (8º E 9) & 1 \\
SEDF & Técnico Em Informática & 1 \\
UCS & Mestrado Profissional Em Ensino De Ciências E Matemática & 1 \\
UEA & Licenciatura Em Computação & 1 \\
UENP & Mestrado Profissional Em Ensino & 1 \\
UENP & Mestrado Profissional Em Educação & 1 \\
UFMT & Graduação Em Tecnologia Educacional Licenciatura & 1 \\
UPF & Programa De Pós-Graduação Em Ensino De Ciências E Matemática & 1 \\
UNESP & $?$ & 1 \\
$?$ & Ciência Da Computação/Análise E Desenvolvimento De Sistemas & 1 \\
$?$ & Computação &
\end{tabular}

Como exibido, em 24 REAs foram mapeados a instituição de ensino e o curso relacionado. Em um recurso apenas a instituição foi identificada e o curso não. Por fim, dois materiais apresentaram o curso, mas não a instituição produtora. Dez materiais não apresentaram informações sobre a sua origem de produção.

\section{Achados e Implicação dos Resultados}

Considerando a falta de informações sobre os REAs para o ensino e aprendizado de programação no Brasil, os resultados deste estudo contribuem para reduzir uma lacuna da literatura atual. Com base na investigação conduzida, coloca-se em perspectiva três achados para a comunidade: Primeiro, cursos de pós-graduação estão produzindo e disponibilizando REAs como produtos de dissertações e/ou de monografias. Isto demonstra que artefatos oriundos das pesquisas realizadas podem ser disponibilizados como produtos educacionais para outros usuários. Segundo, a abertura dos recursos ainda precisa ser repensada entre os produtores. Adotar uma licença permissiva é algo relevante, mas para o REA ser de fato aberto é necessário que a extensão ou formato técnico do material permita adaptação, aumentando a abertura técnica e também implicando na característica do recurso. O mesmo vale para a abertura social, visto que não foi identificada nenhuma informação sobre a acessibilidade do recurso. Terceiro, há desafios no processo de classificação e identificação destes materiais que limitam o seu impacto. 
Com base em tais achados, as principais implicações desta pesquisa estão nos desafios que os usuários e produtores de REAs devem superar. Como apresentado na Seção 3. foi necessário realizar uma série de etapas nas buscas a fim de identificar os recursos para análise. Este não é um problema único da área de programação, a própria UNESCO, que formalizou o termo REA, reconhece que para avançar a cultura dos recursos abertos é necessário o estabelecimento de mecanismos que facilitem a recuperação efetiva dos materiais [Unesco 2019]. No Brasil, além do estabelecimento de mecanismos, é necessário catalogar locais que possuem acervos de programação e propor ferramentas para identificar e recuperar os materiais. Os desafios disso são expostos na dificuldade de encontrar termos relevantes nos materiais, assuntos e níveis educacionais, conforme atestado na $\mathrm{RQ}_{1}$. Tal fragmentação limita o potencial e o impacto dos recursos. Tendo em vista que termos não relevantes e genéricos estão sendo sendo usados, tornando o REA difícil de ser identificado.

Por fim, além dos programas de pós-graduação, os estudos científicos de programação podem se beneficiar do uso de portais ou repositórios abertos para divulgar as ferramentas produzidas. Isso facilitaria a disseminação da pesquisa ao ponto que ajudaria professores e alunos a usarem os produtos gerados. Ademais, essa ação facilitaria a replicabilidade do estudo por outros pesquisadores, tendo em vista que reproduzir uma investigação da área é um desafio devido à ausência dos artefatos [Araujo et al. 2016]. Essa ação simples pode consolidar o avanço da cultura aberta mais rapidamente. A área de programação é historicamente aderente as práticas abertas, tendo em vista o conhecimento e a prática da cultura do open source por professores e alunos. Assim sendo, a comunidade pode fornecer além dos códigos os recursos produzidos como sendo componentes educacionais para outros usuários utilizarem, adaptarem ou redistribuírem.

\section{Considerações Finais}

Este estudo apresentou os primeiros referenciais sobre os REAs voltados ao ensino de programação no Brasil. Os resultados demonstram que o conteúdo, abertura e produção ocorrem, mas ainda existem desafios a serem solucionados. Mesmo assim, tais problemas podem ser superados conforme o avanço e entendimento da própria comunidade. Este estudo é um esforço inicial a fim de compreender o movimento de pesquisadores, docentes e discentes de programação, que produzem, usam e adaptam REAs.

Limitações: As principais limitações deste estudo referem-se ao tamanho amostral e o procedimento de coleta de dados. A amostra analisada representa uma fração do conjunto de REAs voltados ao ensino de programação no Brasil. Para reduzir o risco desta ameaça, foi selecionado um portal educacional com materiais próprios e de outros repositórios brasileiros. Em relação a coleta de dados, devido ao processo de filtragem, pode ser que recursos potencialmente relevantes tenham sido descartados. Para reduzir o risco desta ameaça foram testadas diferentes termos na string de busca e optou-se pela string que retornou o maior número de resultados.

Trabalhos Futuros: Como sugestão de trabalhos futuros, recomenda-se a disponibilização de mecanismos para apoiar a busca e recuperação de REAs voltados ao ensino de programação. A identificação destes materiais ainda é um desafio para a comunidade já que os recursos estão fragmentados e dispersos. Além disso, é necessário focar em práticas abertas a fim de disseminar uma cultura do reuso de materiais educaci- 
onais. Neste sentido, a necessidade de promover na comunidade de alunos e professores de programação a importância da abertura técnica. Ao mesmo tempo, é importante divulgar o uso destes materiais ao invés de recursos fechados ou com direitos autorais para pesquisadores da área de ensino e aprendizado de programação. Por fim, recomenda-se a replicação deste estudo em outras fontes de REAs, usando novas strings de busca, com mais termos de programação.

\section{Agradecimentos}

Este estudo foi parcialmente financiado pela Coordenação de Aperfeiçoamento de Pessoal de Nível Superior - Brasil (CAPES) - Código Financeiro 001 e pela Fundação de Amparo à Pesquisa do Estado de São Paulo (FAPESP 2018/26636-2).

\section{Referências}

Amiel, T., Gonsales, P., and Sebriam, D. (2018). Recursos educacionais abertos no brasil: 10 anos de ativismo. In EmRede - Revista de Educação a Distância, volume 5, page 246-258.

Amiel, T., Mesquita, R., Oddone, A., Alexandre, M., Miguel, G., and Figuerôa, M. (2019). Recursos educacionais e abertura: Percepções e práticas docentes no ensino superior. In Anais do XXV Workshop de Informática na Escola, volume 25, page 879888, Brasília, Brasil.

Araujo, A., Andrade, W., and Guerrero, D. (2016). Um mapeamento sistemático sobre a avaliação do pensamento computacional no brasil. In Anais dos Workshops do Congresso Brasileiro de Informática na Educação, volume 5, page 1147, Uberlândia, Brasil.

Baldwin, D. (2015). Can we flip non-major programming courses yet? In Proceedings of the 46th ACM Technical Symposium on Computer Science Education, page 563-568, Kansas City, United States.

Chan, H. C. B., Ho, Y. H., Tovar, E., and Reisman, S. (2020). Enhancing the learning of computing/it students with open educational resources. In Proceedings of the IEEE 44th Annual Computers, Software, and Applications Conference, page 113-122, Madrid, Spain.

Dichev, C. and Dicheva, D. (2012). Open educational resources in computer science teaching. In Proceedings of the 43rd ACM Technical Symposium on Computer Science Education, page 619-624, Raleigh, United States.

Filho, J. A. C., Freire, R. S., and Maya, D. L. (2021). Estudo de Caso como método de pesquisa em Informática na Educação. Série Metodologia de Pesquisa em Informática na Educação. (v.3).

OECD, O. (2007). Giving knowledge for free: The emergence of open educational resources. http://www.oecd.org/education/ceri/38654317.pdf.

Unesco (2019). Recommendation on open educational resources (OER). https://unesdoc.unesco.org/ark:/48223/pf0000373755/PDF/373755eng.pdf.multi.

Wiley, D. (2014). The access compromise and the 5th R. https://opencontent.org/blog/archives/3221. 